\title{
A Hybrid Recommendation Model for Learning Object Repositories
}

\author{
A. Gordillo, E. Barra and J. Quemada
}

\begin{abstract}
Learning Objects (LOs) have emerged as a cornerstone approach for the development and distribution of educational content. These resources are distributed by Learning Object Repositories (LORs), which can make it easier for users to find suitable LOs by using Recommender Systems (RSs). This paper presents a hybrid recommendation model for LORs that combines content-based, demographic and context-aware techniques, along with the use of quality and popularity metrics. This article also describes how the model has been used to implement two RSs for two real LORs: ViSH and Europeana. Each of these RSs was evaluated in terms of accuracy, utility, usability and satisfaction perceived by end users. Besides, an A/B testing was performed in ViSH to compare the recommendations of the RS with random suggestions. The results showed that the RSs had a high user acceptance in terms of utility, usability and satisfaction, and that the RSs significantly exceeded the performance achieved by the random recommendations.
\end{abstract}

Keywords - Hybrid Recommender Systems, Recommender model, Learning Objects, Learning Object Repositories.

\section{INTRODUCCIÓN}

$\mathrm{D}$ URANTE los últimos años los Objetos de Aprendizaje (OAs) se han consolidado como una estrategia fundamental para el desarrollo y distribución de contenidos educativos. En este trabajo definimos los OAs como "recursos digitales reusables, autónomos y etiquetados con metadatos que pueden ser utilizados para la educación" [1]. La diferencia fundamental de los OAs respecto a otro tipo de recursos educativos es que estos son diseñados con el fin de facilitar la reutilización de contenidos [2], lo cual permite ahorrar tiempo y disminuir costes de producción. Para facilitar la búsqueda y reutilización de los OAs estos se etiquetan con metadatos que incluyen información como el título, descripción, idioma o fecha de creación del recurso. Esta información suele ser guardada de forma estructurada siguiendo algún estándar como LOM [3] o Dublin Core [4]. Los beneficios de usar OAs en diferentes entornos educativos han sido estudiados y comprobados en numerosas investigaciones [5]-[7].

Los Repositorios de Objetos de Aprendizaje (ROAs) son sistemas web que almacenan, clasifican y distribuyen recursos educativos en forma de OAs. Además de proporcionar un servicio de búsqueda de OAs, algunos ROAs ofrecen servicios adicionales que permiten a los usuarios la creación de una cuenta personal, publicar recursos, crear contenidos, usar catálogos, clasificar los OAs en carpetas personales o participar en foros entre otras posibilidades [8], [9]. Entre los
ROAs más populares podemos destacar MERLOT, AGREGA, ARIADNE o Connexions. La cantidad de recursos disponibles en los ROA se ha disparado en los últimos años, provocando que los usuarios tengan grandes dificultades para encontrar contenidos relevantes y de calidad. Esta dificultad ha sido puesta de manifiesto por algunos estudios que han reportado que los profesores perciben este proceso de búsqueda como costoso en tiempo e incluso frustrante, pudiendo llegar a ser necesaria más de una hora para encontrar un recurso adecuado [10]. Para tratar de solventar este problema los ROAs están adoptando, tanto de forma aislada como conjunta, diversas medidas como la mejora de los servicios de búsqueda mediante la adopción de nuevas métricas de ranking [11], la implementación de mecanismos de control de calidad [12] o el desarrollo de Sistemas de Recomendación (SRs) [13]-[16].

Existen numerosas investigaciones recientes sobre la utilización de SRs en entornos virtuales de aprendizaje para facilitar a los usuarios la búsqueda de recursos educativos en base a sus necesidades y preferencias [17]. No obstante y a pesar de la cantidad de sistemas propuestos, un análisis exhaustivo de la situación actual del desarrollo de estos SRs revela que la mayoría de ellos se encuentran todavía en fase de diseño o prototipado, que pocos han sido evaluados con usuarios humanos y que existe una notable carencia de estudios de evaluación en contextos reales [18]. Esta carencia se debe principalmente a las dificultades que presenta la implementación de un SR en un escenario real frente a un prototipo [19]. Cuando se implementa un SR para un escenario real el entorno juega un papel crucial. A este respecto, [19] propone estudiar el entorno en base a tres dimensiones: usuarios, datos utilizados para generar las recomendaciones y finalmente la aplicación global en la que se va a integrar el SR. Otra carencia importante en la literatura además de la evaluación de SRs en escenarios reales, es la evaluación de un mismo modelo en distintos escenarios.

Este artículo presenta un modelo de recomendación híbrida para ROAs, así como la implementación y evaluación de dos SRs basados en dicho modelo y aplicados a dos ROAs reales: ViSH y Europeana. Para cada uno de estos SRs se evaluó su precisión y utilidad así como la usabilidad y satisfacción percibida por los usuarios finales. Adicionalmente, se realizó un test A/B en ViSH para evaluar el SR en el contexto real.

El resto del artículo se estructura de la siguiente forma. La siguiente sección introduce los SRs y su aplicación en el campo del aprendizaje potenciado por la tecnología. La sección 3 presenta el modelo de recomendación para ROAs. La sección 4 describe los SRs implementados para ViSH y Europeana y la 5 expone sus respectivas evaluaciones. Finalmente, la última sección resume las conclusiones y propone líneas de investigación futuras. 


\section{SISTEMAS DE RECOMENDACIÓN}

Los Sistemas de Recomendación (SRs) son herramientas software que generan sugerencias de objetos que son de probable interés o relevancia para un usuario. En función de las técnicas utilizadas para generar las recomendaciones podemos distinguir entre diferentes tipos de SRs ([20], [21]):

- Basados en contenido: el sistema aprende a recomendar objetos similares a los que le gustaron o interesaron al usuario en el pasado. Las recomendaciones son generadas en base a las características de los objetos y a las calificaciones y/o acciones del usuario.

- Filtrado colaborativo: recomiendan al usuario objetos que les gustaron o interesaron en el pasado a otros usuarios con gustos similares. La similitud en el gusto de dos usuarios se calcula en base a la similitud de sus históricos de calificaciones.

- Demográficos: este tipo de sistemas recomiendan objetos basándose en el perfil demográfico del usuario: idioma, país, edad, género, etc.

- Basados en conocimiento: estos SRs sugieren objetos en base a inferencias sobre las preferencias $y$ necesidades del usuario. Para ello suelen utilizar conocimientos específicos del dominio sobre cómo ciertas características de los objetos cubren las necesidades de los usuarios.

- Basados en comunidad (social): el sistema realiza recomendaciones basándose en las preferencias de los amigos o contactos del usuario. Para ello hace uso de la información sobre las relaciones sociales del usuario y las preferencias de sus contactos.

- Hibridos: son SRs que combinan dos o más de las técnicas anteriores para mejorar las recomendaciones. Este tipo de SR puede ser a su vez clasificado en función del tipo de combinación utilizada [21], [22].

Otro tipo de SR son los llamados "sensibles al contexto", los cuales incorporan información contextual en el proceso de recomendación ([20], [23]). Esta información contextual puede incluir diferentes dimensiones tales como la hora, localización geográfica o actividad actual, o el dispositivo utilizado. En el ámbito educativo, los SRs suelen tomar en cuenta recursos digitales relevantes para el usuario en la información contextual [23]. Un caso típico es recomendar objetos similares a aquellos que el usuario está viendo.

Los SRs también se pueden clasificar entre proactivos y reactivos. Los SRs proactivos se diferencian de los reactivos en que no requieren de acciones ni peticiones explícitas de los usuarios para generar las recomendaciones.

En el campo del aprendizaje potenciado por la tecnología, los SRs se utilizan principalmente para ayudar a estudiantes y profesores a encontrar recursos educativos, pero en menor medida también para recomendar secuencias de recursos, sugerir compañeros o proponer actividades didácticas [17]. En los últimos años se han publicado varios trabajos sobre SRs para ROAs. Cechinel et al. [15] evaluaron recomendaciones de recursos educativos de MERLOT generadas por diferentes algoritmos de filtrado colaborativo. Fraihat y Shambour [13] presentaron un SR semántico reactivo para asistir a los estudiantes en la búsqueda de OAs. Otro ejemplo de sistema reactivo para asistir a estudiantes en las búsquedas dentro un ROA puede encontrarse en [16], implementado en este caso como un SR híbrido que combina técnicas colaborativas, basadas en contenido y demográficas. Cubriendo el mismo caso de uso, [14] describe un modelo de recomendación reactiva basada en contenido para ROAs que prioriza aquellos recursos que son similares a las consultas de los estudiantes y que al mismo tiempo presentan mayor utilidad pedagógica. También existen trabajos sobre recomendación en ViSH y Europeana. Por ejemplo, [24] describe un modelo teórico para implementar un recomendador social proactivo sensible al contexto en ViSH. Un estudio posterior determinó algunos parámetros del modelo teórico mediante una encuesta a más de 100 usuarios [25]. El modelo nunca llegó a implementarse por completo y carece de evaluaciones de precisión, utilidad o aceptación. Clough et al. [26] diseñaron un modelo para producir sugerencias no personalizadas (sin tener en cuenta al usuario) de recursos de Europeana utilizando una técnica basada en contenido que calcula la similitud en base a los metadatos. El modelo solo fue implementado mediante un prototipo que no fue evaluado. Más adelante, [27] estudió el uso de algoritmos de PageRank personalizado para generar recomendaciones de recursos de Europeana. La evaluación se basó en registros de búsqueda sin involucrar a usuarios reales.

La utilidad de los SRs no se limita a los ROAs, su uso también ha sido estudiado en otros escenarios como sistemas de gestión del aprendizaje [28], ambientes de aprendizaje ubicuo ([29], [30]), repositorios de analíticas de aprendizaje [31] o portales web de instituciones educativas [32]. En la revisión publicada recientemente por Drachsler et al. [17] se analizan y clasifican 82 SRs aplicados al campo del aprendizaje potenciado por la tecnología en diferentes entornos.

\section{MODELO DE RECOMENDACIÓN}

El esquema del modelo de recomendación para ROAs se representa en la Fig. 1. Se trata de un modelo híbrido que combina técnicas de recomendación basada en contenido, demográfica y sensible al contexto, junto con el uso de métricas de calidad y popularidad. El modelo permite generar recomendaciones proactivas de conjuntos de $\mathrm{N}$ objetos.

\section{A. Casos de uso}

El modelo se diseñó para soportar una amplia variedad de casos de uso considerando las actividades y servicios más habituales de los ROAs. Otro factor que se tuvo en cuenta fue los accesos por parte de usuarios anónimos (usuarios no registrados o que no han iniciado sesión) carentes de perfil de usuario. Este es un factor importante en los ROAs ya que generalmente se produce una gran cantidad de accesos de usuarios anónimos. Nuestra experiencia con ViSH nos ha permitido comprobar que en torno al $80 \%$ de los accesos son realizados por usuarios no identificados. A continuación se describen los principales casos de uso soportados. 


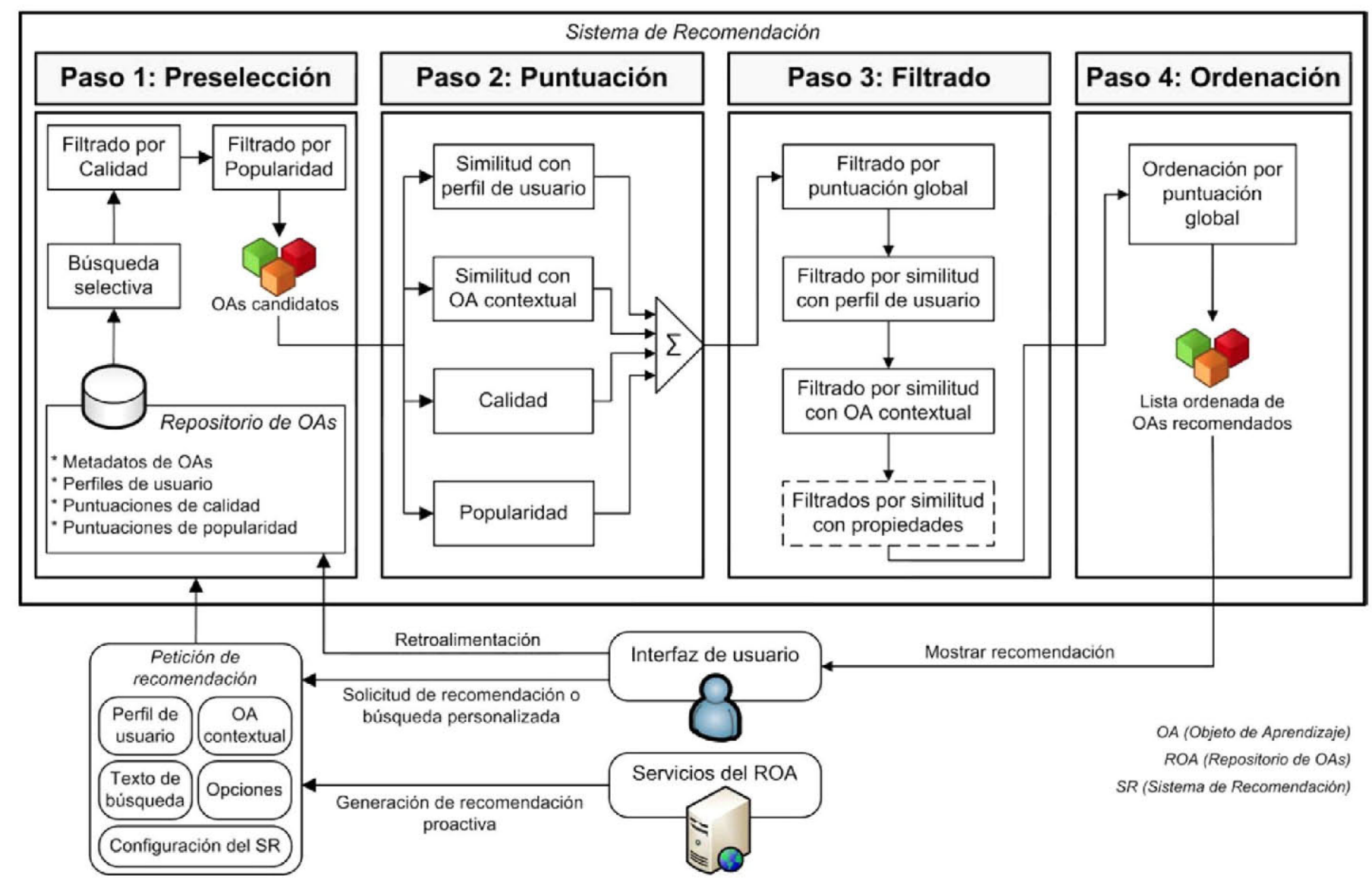

Figura 1. Modelo de Recomendación Híbrida para Repositorios de Objetos de Aprendizaje (ROAs).

\section{1) Recomendar una lista de OAs a un usuario particular}

Es el caso de uso más habitual, resulta útil para mostrar recomendaciones en la página principal, la página de perfil del usuario o en situaciones en las que se carezca de información contextual. Para un usuario anónimo las recomendaciones son generadas en base a las puntuaciones de calidad y popularidad. Si el usuario ha iniciado sesión puede utilizarse además su perfil. Para los usuarios anónimos existe la posibilidad de generar un perfil de usuario virtual a lo largo de la sesión.

\section{2) Recomendar a un usuario OAs similares a otro}

Aunque algunos ROAs solo muestran metadatos, muchos otros permiten al usuario ver los OAs e interactuar con ellos. Recomendar OAs similares a aquellos que el usuario está viendo es otro caso de uso típico. En estas situaciones, el SR puede valerse del OA que el usuario está viendo (al que hemos llamado $O A$ contextual en el modelo) para generar las recomendaciones. Tanto al acceder a la página del OA como tras finalizar el visionado son momentos apropiados para mostrar estas recomendaciones [25]. Cabe destacar que para que un SR pueda generar este tipo de recomendaciones debe ser sensible al contexto, ya que necesita conocer tanto la actividad realizada por el usuario (ver un OA) como el OA contextual. En este caso, el modelo genera las sugerencias en base a la similitud con el OA contextual, las puntuaciones de calidad y popularidad, y el perfil de usuario en caso de que se trate de un usuario identificado.

\section{B. Entidades}

Esta sección describe las diferentes entidades contempladas por el modelo en el proceso de recomendación.

\section{1) Objetos de aprendizaje}

El SR utiliza propiedades incluidas en los metadatos para modelar los OAs. Las propiedades concretas a utilizar dependerán del entorno donde opere el SR. Algunas de las más habituales, contempladas por el estándar LOM [3] son título, descripción, idioma y palabras clave. Los ROAs pueden usar perfiles de aplicación para extender LOM o estructuras de metadatos diferentes habilitando nuevas propiedades. El modelo permite usar cualquier propiedad de tipo texto (título, descripción), número o fecha (fecha de creación), vocabulario (categoría en un catálogo, idioma) o listas de elementos de tipo vocabulario (palabras clave). Los elementos de tipo vocabulario son aquellos que pertenecen a una lista de valores, generalmente procedente de una taxonomía.

\section{2) Perfiles de usuario}

Los usuarios se modelan mediante diferentes propiedades. Por un lado con datos demográficos como el idioma o la edad. Por otro lado con información explícita que pueda dar el usuario sobre sus gustos (áreas de interés). Por último, el perfil de usuario puede incluir los OAs que le gustaron o interesaron al usuario en el pasado, así como los que no lo hicieron. Los OAs se guardan acorde al modelo anterior y pueden ir 
acompañados de una calificación numérica o simplemente de un dato binario que indique el interés positivo o negativo del usuario. Las propiedades concretas a utilizar así como el modo de generar el perfil dependerán del entorno. Un ROA puede optar por obtener los OAs de interés para un usuario mediante valoraciones, con un sistema de favoritos o considerando los OAs visitados durante la sesión. El modelo da libertad al ROA para usar cualquiera de estas estrategias, incluso para elegirlas en función del perfil del usuario.

\section{3) Puntuaciones de calidad}

Las métricas de calidad tienen como objetivo medir y cuantificar numéricamente la calidad de los OAs. E1 uso de estas métricas permite calcular puntuaciones de calidad. Existen diversos modelos de evaluación de OAs con diferentes conceptos de calidad, algunos evalúan solo la calidad de los metadatos o la reusabilidad, mientras que otros como LORI [33] o la escala WBLT [34] evalúan el OA al completo incluyendo una valoración del contenido desde un punto de vista pedagógico. En los ROAs se suelen emplear modelos de evaluación pedagógicos donde las puntuaciones de calidad son obtenidas a partir de valoraciones de usuarios o revisores. Las métricas de calidad basadas en LORI se han mostrado fiables para ordenar resultados de búsqueda en ROAs [35]. El modelo de recomendación propuesto da libertad al ROA para elegir la métrica de calidad a utilizar, siendo la única condición que las puntuaciones estén en una escala de $0-1$.

\section{4) Puntuaciones de popularidad}

Las métricas de popularidad pretenden medir el grado de uso de los OAs y suelen estar basadas en parámetros de uso tales como el número de visitas o la cantidad de descargas. Hay estudios sobre el uso de estas métricas en herramientas de búsqueda de ROAs [11] y acerca de su integración con métricas de calidad [36]. El modelo no impone restricción alguna al ROA sobre qué métrica utilizar, siendo la única condición que esta calcule la popularidad de los OAs en una escala de $0-1$.

\section{Proceso de recomendación}

El proceso de recomendación se compone de las siguientes etapas representadas en la Fig. 1.

\section{1) Petición de recomendación}

El proceso comienza con una petición que puede ser solicitada por el usuario o de forma proactiva. El ROA indica en la petición el perfil del usuario (en caso de existir), el OA contextual (para el caso de uso 2), y una serie de opciones, por ejemplo, el número de recomendaciones deseadas (N). Si se desea utilizar el SR para realizar una búsqueda personalizada la petición debe incluir además el texto de búsqueda. La petición también puede contener ajustes de configuración para el SR, como los pesos a usar en el paso 2 o los umbrales de los filtros. La idea es que el SR tenga una configuración por defecto, pero que el modelo sea lo suficientemente flexible para permitir a los usuarios de los ROAs modificar esta configuración, bien de forma permanente (ajustes en su perfil) o bien para una petición aislada.

\section{2) Paso 1: Preselección}

En un ROA con ingentes cantidades de recursos no resulta viable procesarlos todos en cada petición. El objetivo de este paso es seleccionar de la base de datos un conjunto de OAs candidatos que pueda ser procesado de forma ágil por los siguientes pasos. Si se han especificado términos de búsqueda el SR obtendrá un conjunto inicial de OAs que encaje con ellos, al estilo de un motor de búsqueda tradicional. En esta búsqueda selectiva de OAs, además de filtros de calidad y popularidad, se pueden imponer una serie de filtros de preselección. Los filtros concretos a utilizar dependerán del escenario particular del ROA. Por ejemplo, un ROA podría optar por recomendar únicamente OAs en el idioma del usuario. Los OAs candidatos entregados a la salida son un conjunto de OAs acordes a los criterios de preselección (idioma, calidad mínima, etc.), cuyo número es igual o inferior a un tamaño máximo de preselección establecido por el ROA. Tras el proceso de filtrado puede ocurrir que la cantidad de OAs sea superior o inferior al máximo permitido. $\mathrm{Si}$ es superior se elegirán los candidatos finales al azar (introduciendo aleatoriedad a las recomendaciones). Si resulta menor, el ROA puede elegir entre no hace nada o rellenar el conjunto con otros OAs siguiendo criterios menos restrictivos. Dependiendo del escenario, puede interesar que el usuario reciba siempre recomendaciones al margen de su idoneidad.

\section{3) Paso 2: Puntuación}

Este paso calcula para cada OA candidato sus puntuaciones parciales y su puntuación global. A mayor puntuación global, mayor probabilidad tiene un $\mathrm{OA}$ de ser recomendado. Primero se calcula una puntuación parcial por cada criterio de recomendación: similitud con perfil de usuario, similitud con el OA contextual, calidad y popularidad. No es obligatorio el procesamiento de todos los bloques. Para usuarios anónimos se omitirá el bloque de similitud con perfil de usuario, cuando no haya OA contextual se saltará el segundo bloque, y en el caso de que un ROA no dispusiera de métricas de calidad y/o popularidad también podrían omitirse esos bloques. Las métricas empleadas para calcular las puntuaciones parciales se describen más adelante. Por último, la puntuación global de cada OA se obtiene como una media aritmética ponderada de las puntuaciones parciales. La importancia de cada criterio de recomendación se ajusta mediante los pesos de esta operación, que pueden tener valores fijos establecidos por el ROA o configurados por el usuario. Todas las puntuaciones, incluida la global, se obtienen en una escala de 0-1.

\section{4) Paso 3: Filtrado}

Aquí el conjunto de OAs se filtra en base a las puntuaciones anteriores. Se pueden descartar OAs que no hayan alcanzado un determinado umbral de puntuación global así como OAs que no tengan una mínima similitud con el perfil de usuario o el OA contextual. También es posible establecer filtros para propiedades específicas, por ejemplo, para descartar todos los OAs cuya descripción tenga una similitud con la del OA contextual inferior a un determinado valor. Al igual que en el paso 2, solo se procesan los bloques que apliquen al caso de 
uso. Es decir, si no existe OA contextual, todos los filtros que hagan uso de él no serán aplicados. Los filtros a utilizar en el SR así como sus umbrales por defecto (ajustables por los usuarios) dependerán del entorno concreto del ROA.

\section{5) Paso 4: Ordenación}

Finalmente, el SR ordena los OAs candidatos de acuerdo a su puntuación global y genera la recomendación final como una lista de los $\mathrm{N}$ primeros, donde $\mathrm{N}$ el número de recomendaciones deseadas especificado en la petición.

\section{6) Visualización de la recomendación}

En esta última etapa la lista de OAs recomendados generada en el paso anterior es mostrada en la interfaz de usuario. El modelo no restringe la forma de mostrar las recomendaciones. Finalmente, es posible hacer uso de la retroalimentación del usuario para mejorar las recomendaciones futuras. Esta retroalimentación puede darse de diversas maneras: aceptar o rechazar una recomendación, evaluar un OA (calidad), visitar o descargar un OA (popularidad), marcar un OA como favorito (declaración explícita de interés), interactuar con un OA (analíticas de aprendizaje), etc. También existe la posibilidad de que los usuarios definan de forma explícita sus intereses en su perfil de usuario.

\section{Métricas de similitud}

A continuación se exponen las métricas utilizadas para calcular las diferentes similitudes. Todos los pesos y parámetros pueden ser ajustados en función del entorno.

1) $O A-O A$

Esta métrica calcula la similitud entre dos OAs en base a sus propiedades de acuerdo a la siguiente expresión:

$$
\operatorname{Sim}_{O A-O A}\left(O A_{x}, O A_{y}\right)=\sum_{i=1}^{N} W p_{i} \times \operatorname{Sim}_{p}\left(O A x_{i}, O A y_{i}\right)
$$

Donde $\mathrm{N}$ es el número de propiedades de los $\mathrm{OAs}, \mathrm{Wp}_{\mathrm{i}}$ es el peso asignado a la propiedad i-ésima cumpliéndose además que la suma de todos los pesos es igual a $1, \mathrm{OAx}_{\mathrm{i}} \mathrm{y} \mathrm{OAy}_{\mathrm{i}}$ son respectivamente las propiedades i-ésimas de $\mathrm{OA}_{\mathrm{x}}$ y $\mathrm{OA}_{\mathrm{y}}, \mathrm{y}$ $\operatorname{Sim}_{\mathrm{p}}$ es una función concreta que calcula la similitud entre $\mathrm{OAx}_{\mathrm{i}}$ y $\mathrm{OAy}_{\mathrm{i}}$ en una escala de 0-1. La función $\operatorname{Sim}_{\mathrm{p}}$ a utilizar en cada caso depende del tipo de propiedad. Los apartados 2-5 detallan las diferentes funciones $\operatorname{Sim}_{\mathrm{p}}$ disponibles.

\section{2) Texto}

Para calcular la similitud entre dos textos se emplea la métrica similitud coseno en combinación con la medida TF-IDF (Term Frequency - Inverse Document Frequency) [37], de acuerdo a la siguiente expresión:

$$
\begin{gathered}
\operatorname{Sim}_{\text {texto }}\left(T_{x}, T_{y}\right)= \\
\frac{\sum_{i=1}^{N} T F-I D F\left(P_{i}, T_{x}\right) \times T F-I D F\left(P_{i}, T_{y}\right)}{\sqrt{\sum_{i=1}^{N} T F-I D F\left(P_{i}, T_{x}\right)^{2}} \times \sqrt{\sum_{i=1}^{N} T F-I D F\left(P_{i}, T_{y}\right)^{2}}}
\end{gathered}
$$

Donde $\mathrm{N}$ es la cantidad de palabras (o términos) diferentes de los textos $\mathrm{T}_{\mathrm{x}}$ y $\mathrm{T}_{\mathrm{y}}, \mathrm{P}_{1}$ es la palabra i-ésima, y la función $\mathrm{TF}-\mathrm{IDF}(\mathrm{P}, \mathrm{T})$ se define de la siguiente forma:

$$
T F-I D F(P, T)=T F(P, T) \times I D F(P)
$$

Donde $\mathrm{TF}(\mathrm{P}, \mathrm{T})$ es el número de ocurrencias de la palabra $\mathrm{P}$ en el texto $\mathrm{T}$, y la función $\operatorname{IDF}(\mathrm{P})$ es:

$$
I D F(P)=\log \frac{N}{1+N_{P}}
$$

Siendo $\mathrm{N}$ la cantidad total de OAs del repositorio, y $\mathrm{N}_{\mathrm{p}}$ el número de OAs del repositorio cuyas propiedades textuales incluyen la palabra $P$.

\section{3) Vocabulario}

La similitud de dos propiedades de tipo vocabulario será 1 si ambas tienen el mismo valor y 0 en caso contrario.

\section{4) Números y fechas}

La similitud entre dos propiedades numéricas con valores $\mathrm{N}_{\mathrm{x}}$ y $\mathrm{N}_{\mathrm{y}}$ se puede calcular acorde a la siguiente expresión:

$$
\operatorname{Sim}_{\text {numérica }}\left(N_{x}, N_{y}\right)=\left(1-\frac{\left|N_{x}-N_{y}\right|}{E_{\text {Max }}-E_{\text {Min }}}\right)^{2}
$$

Siendo $\mathrm{E}_{\mathrm{Max}} \mathrm{y} \mathrm{E}_{\mathrm{Min}}$, respectivamente, el máximo y mínimo de la escala considerada, y cumpliendo que $\mathrm{N}_{\mathrm{x}}, \mathrm{N}_{\mathrm{y}} \in\left[\mathrm{E}_{\mathrm{Min}}, \mathrm{E}_{\mathrm{Max}}\right]$. Esta métrica también sirve para fechas ya que estas pueden ser convertidas a números fácilmente.

\section{5) Listas de elementos de tipo vocabulario}

La similitud entre dos listas de elementos de tipo vocabulario viene dada por la siguiente expresión:

$$
\operatorname{Sim}_{\text {listas }}\left(L_{x}, L_{y}\right)=\frac{2 \times c}{d_{x}+d_{y}}
$$

Donde $\mathrm{c}$ es el número de elementos comunes entre las listas, $\mathrm{y}$ $\mathrm{d}_{\mathrm{x}}, \mathrm{d}_{\mathrm{y}}$ son, respectivamente, los tamaños de las listas $\mathrm{L}_{\mathrm{x}} \mathrm{y} \mathrm{L}_{\mathrm{y}}$.

\section{6) Perfil de Usuario - $O A$}

Esta métrica calcula la similitud entre un usuario y un OA en base al perfil del usuario y las propiedades del OA conforme a la siguiente función:

$$
\begin{gathered}
\operatorname{Sim}_{U-O A}(U, O A)= \\
W p_{1} \times \operatorname{Sim}_{O A-O A S}\left(O A, U_{1}\right)+\sum_{i=2}^{N} W p_{i} \times \operatorname{Sim}_{p}\left(U_{i}, O A_{i}\right)
\end{gathered}
$$

Donde $\mathrm{N}$ es el número de propiedades con las que se ha modelado el perfil de usuario $U, U_{i}$ es la propiedad i-ésima de $\mathrm{U}, \mathrm{W} \mathrm{p}_{1}$ es el peso asignado a $\mathrm{U}_{\mathrm{i}}$ cumpliéndose además que la suma de todos los pesos es igual a $1, \mathrm{y}_{1}$ (con peso $\mathrm{Wp}_{1}$ ) es la lista de OAs almacenada en el perfil de usuario. $\mathrm{OA}_{i}$ es la propiedad del OA relacionada con $\mathrm{U}_{\mathrm{i}}, \operatorname{Sim}_{\mathrm{p}}$ es una función que calcula la similitud entre las propiedades $U_{i}$ y $O_{i}$ en una escala de 0-1 de la misma forma que en la función $\operatorname{Sim}_{\mathrm{OA}-\mathrm{OA}}, \mathrm{y}$ la función $\operatorname{Sim}_{\mathrm{OA}-\mathrm{OAs}}$ es una función que calcula la similitud entre un OA y una lista de OAs de la siguiente forma:

$$
\operatorname{Sim}_{O A-O A s}(O A, O A s)=\frac{\sum_{i=1}^{N} \alpha_{i} \times \operatorname{Sim}_{O A-O A}\left(O A, O A s_{i}\right)}{\sum_{i=1}^{N}\left|\alpha_{i}\right|}
$$

Siendo $\mathrm{N}$ el tamaño de la lista OAs, $\mathrm{OAs}_{\mathrm{i}}$ el OA i-ésimo de la lista OAs, y $\alpha_{i}$ el peso de OAs $s_{i}$. El parámetro $\alpha_{i}$ representa cuanto le gusta o interesa $\mathrm{OAs}_{\mathrm{i}}$ al usuario. Puede ser binario o tomar un rango de valores en función de cómo se capturen las preferencias de los usuarios en el ROA. Incluso puede ser negativo si se quiere penalizar OAs similares a otros 
rechazados, aunque en este caso la métrica arrojaría valores en el intervalo $[-1,1]$ en lugar de $[0,1]$.

Procesar todos los OAs almacenados en el perfil de usuario resultaría inviable en muchos casos si se quieren generar recomendaciones en tiempo real en base a las acciones del usuario durante la sesión. El modelo contempla esta opción permitiendo el uso de un subconjunto de OAs. En este caso se emplearía la métrica (7), con $U_{1}$ siendo una lista formada por M OAs elegidos aleatoriamente entre los últimos $\mathrm{N}$ almacenados en el perfil de usuario. Tanto $\mathrm{M}$ como $\mathrm{N}$ son parámetros configurables del SR.

Para calcular la similitud Usuario-OA se deben emplear propiedades de los OAs relacionadas con las del usuario. El ROA debe elegir estas relaciones. Habitualmente se calculan similitudes entre el idioma del usuario y el del contenido del $\mathrm{OA}$, entre la edad del usuario y el nivel educativo o rango de edad recomendado en el OA, y/o entre las áreas de interés del usuario y las palabras clave del OA.

\section{IV.IMPLEMENTACIÓN}

Siguiendo el modelo presentado, se han implementado dos SRs para dos ROAs distintos. Esta sección describe dichas implementaciones así como los diferentes escenarios.

\section{A. $\mathrm{ViSH}$}

ViSH (http://vishub.org) es una plataforma de e-Learning centrada en la creación y compartición de recursos educativos abiertos formada por un ROA, una red social y herramientas de creación de contenidos [38]. Los usuarios de ViSH pueden publicar contenidos tanto subiendo recursos como a través de herramientas web disponibles en la plataforma. El recurso estrella son las Presentaciones Interactivas, unos OAs creados por los usuarios de ViSH mediante una herramienta web [39]. Estas presentaciones consisten en una serie de diapositivas que integran diversos contenidos: texto, imágenes, cuestionarios, vídeos, audios, aplicaciones web, etc. La Fig. 2 muestra a modo de ejemplo una de ellas. La búsqueda, uso y descarga de recursos es totalmente libre, los usuarios solo necesitan registrarse para publicar contenidos, o para hacer uso de servicios adicionales como la lista de favoritos. Tanto ViSH como su SR son software libre y su código se encuentra disponible en http://github.com/ging/vish.

\section{1) Casos de uso}

En este escenario existen principalmente tres situaciones en las que se muestran recomendaciones. Cuando los usuarios de ViSH acceden a su página de inicio se muestra una sección con sugerencias de presentaciones. Cuando un usuario accede a un OA, se muestran recomendaciones en la banda lateral derecha tal y como puede verse en la Fig. 2. Finalmente, cuando un usuario llega a la última diapositiva de una presentación, puede solicitar recomendaciones pulsando el botón de 'Siguiente diapositiva'. En este caso, aparece un nuevo panel mostrando seis sugerencias. La primera situación solo puede ocurrir para usuarios identificados mientras que las otras pueden darse también para usuarios anónimos.

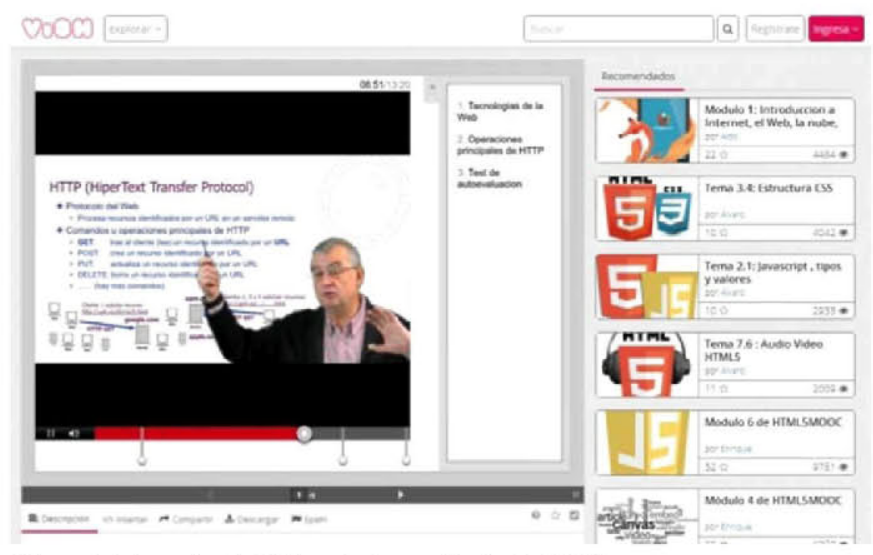

Figura 2. Ejemplo de Objeto de Aprendizaje de ViSH.

\section{2) Objetos de aprendizaje}

Todos los OAs de ViSH están etiquetados con metadatos conformes al estándar LOM. De cara al SR, estos OAs se modelaron mediante las propiedades título (tipo texto, peso $0,2)$, descripción (texto, 0,1), idioma (vocabulario, 0,5) y palabras clave (lista, 0,2).

\section{3) Perfiles de usuario}

Los usuarios se modelaron mediante su idioma (tipo vocabulario, peso 0,25 ), áreas de interés (lista, 0,25$)$ y una lista con los OAs que les interesaron en el pasado (peso 0,5). Esta lista de OAs se va actualizando automáticamente a medida que el usuario accede a recursos durante la sesión. El SR utiliza 1 OA aleatorio de los últimos 4.

\section{4) Puntuaciones de calidad}

ViSH cuenta con un sistema de control de calidad que permite obtener evaluaciones de dos fuentes diferentes. Por un lado, los usuarios pueden evaluar presentaciones empleando las escalas WBLT [34]. Por otro lado, ViSH cuenta con un equipo de revisores que evalúan las presentaciones mediante LORI [33]. En base a estas evaluaciones ViSH calcula puntuaciones de calidad mediante diferentes métricas:

$$
Q_{u}\left(\left\{i_{1}, \ldots, i_{13}\right\}\right)=\frac{5}{3} \times \sum_{i=1}^{13} W_{i} \times\left(i_{i}-1\right)
$$

Siendo $\mathrm{Q}_{\mathrm{u}}$ la calidad asignada por los usuarios en una escala $0-10, \mathrm{i}_{\mathrm{i}}$ la puntuación media dada al criterio i-ésimo del modelo WBLT en una escala 1-7, y W $\mathrm{W}_{\mathrm{i}}$ el peso de ese criterio.

$$
Q_{r}\left(\left\{i_{1}, \ldots, i_{9}\right\}\right)=\frac{5}{2} \times \sum_{i=1}^{9} W_{i} \times\left(i_{i}-1\right)
$$

Siendo $\mathrm{Q}_{\mathrm{r}}$ la calidad asignada por los revisores en una escala $0-10, \mathrm{i}_{\mathrm{i}}$ la puntuación media dada al criterio i-ésimo de LORI en una escala 1-5, y $\mathrm{W}_{\mathrm{i}}$ el peso asignado a ese criterio.

Todos los pesos involucrados en estas métricas fueron obtenidos mediante una encuesta al equipo de revisores de ViSH [35], y cumplen que su suma es igual a 1.

Finalmente, la puntuación global de calidad en escala 0-1 utilizada por el SR de ViSH se obtiene de la siguiente manera:

$$
Q\left(Q_{u}, Q_{r}\right)=\frac{1}{10} \times\left(Q_{u} \times 0,4+Q_{r} \times 0,6\right)
$$

Si un OA no dispone de evaluaciones su puntuación toma un valor provisional de 5 hasta que se produzca la primera. De 
esta forma los OAs recién publicados son beneficiados frente a los OAs que han recibido malas críticas sin dejar de promocionar aquellos con altas valoraciones.

\section{5) Puntuaciones de popularidad}

Para cada presentación, ViSH calcula una puntuación de popularidad en una escala $0-1$ con la siguiente métrica:

$P\left(f_{v}, f_{d}, f_{f}\right)=\frac{f_{v}}{f_{v M A X}} \times 0,4+\frac{f_{d}}{f_{d M A X}} \times 0,1+\frac{f_{f}}{f_{f M A X}} \times 0,5$

Donde $f_{v}$ es la frecuencia mensual de visitas, $f_{d}$ la frecuencia mensual de descargas y $\mathrm{f}_{\mathrm{f}}$ el número medio de usuarios mensuales que añaden el recurso a favoritos. Los parámetros $f_{\mathrm{VMAX}}, \mathrm{f}_{\mathrm{dMAX}} \mathrm{y} \mathrm{f}_{\mathrm{fMAX}}$, son las respectivas frecuencias máximas alcanzadas por un OA en el repositorio.

\section{6) Proceso de recomendación}

En el paso de preselección se establece un filtro de calidad con umbral $0,4 \mathrm{y}$ dos filtros de preselección: idioma y tipo de recurso. El filtro de idioma restringe las recomendaciones a OAs cuyo contenido esté o bien en un idioma conocido para el usuario o bien en el mismo idioma que el OA contextual. El filtro tipo de recurso garantiza que cuando el OA contextual sea una presentación interactiva solo se recomendarán otras presentaciones. Si el OA contextual es de otro tipo se podrá recomendar cualquier recurso relacionado. De este modo, si un alumno español está interactuando con una presentación en inglés recibirá recomendaciones de otras presentaciones en español o inglés cuyas valoraciones no sean inferiores a 4/10. Otra singularidad es que cuando existe un OA contextual, se añaden aleatoriamente a la preselección otros OAs creados por el mismo autor.

En el paso 2 se utilizan los pesos mostrados en la Tabla I. Como se puede apreciar, los pesos variarán en función de si el acceso es realizado por un usuario identificado o no y de la existencia de un OA contextual. En el paso 3 no se aplican filtros adicionales al de calidad, el usuario siempre recibirá recomendaciones independientemente de su idoneidad.

Las recomendaciones se muestran de diferentes maneras, siendo la más habitual la mostrada en la Fig. 2. En ViSH, un usuario puede dar retroalimentación al sistema evaluando las presentaciones siguiendo el modelo WBLT [34] (generando puntuaciones de calidad) y visitando, descargando o marcando como favoritos recursos (alterando la popularidad). Además, cuando un usuario interactúa con un OA este pasa a formar parte de su perfil de usuario automáticamente.

TABLA I

PESOS DEL SISTEMA DE RECOMENDACIÓN DE VISH

\begin{tabular}{|l|c|c|c|c|}
\hline \multirow{2}{*}{$\begin{array}{c}\text { Criterios de } \\
\text { recomendación }\end{array}$} & \multicolumn{4}{|c|}{ Pesos usados en ViSH } \\
\cline { 2 - 5 } & Con $O A$ & Sin $O A$ & Con $O A$ & Sin $O A$ \\
\hline $\begin{array}{l}\text { Similitud con perfil } \\
\text { de usuario }\end{array}$ & 0,2 & 0,8 & - & - \\
\hline $\begin{array}{l}\text { Similitud con OA } \\
\text { contextual }\end{array}$ & 0,6 & - & 0,8 & - \\
\hline Calidad & 0,1 & 0,1 & 0,1 & 0,5 \\
\hline Popularidad & 0,1 & 0,1 & 0,1 & 0,5 \\
\hline
\end{tabular}

\section{B. Europeana}

Europeana es una organización que tiene como objetivo proporcionar acceso a millones de contenidos digitalizados sobre el patrimonio científico y cultural europeo a través de su portal web (http://www.europeana.eu) [40]. Actualmente este portal da acceso a más de $\mathbf{4 5}$ millones de recursos tales como libros, periódicos, revistas, pinturas, música o películas. La organización pretende aumentar el uso de estos recursos en el área educativa durante los próximos años [41]. Europeana no almacena los recursos sino que el portal muestra información incluida en los metadatos y enlaces al contenido. La búsqueda es libre pero los usuarios deben registrarse si quieren tener una lista de favoritos u otros servicios extra. Europeana también ofrece servicios abiertos en forma de APIs para permitir a aplicaciones externas acceder a sus colecciones digitales.

En este escenario el SR se ha implementado como una aplicación independiente capaz de generar recomendaciones de OAs obtenidos de Europeana a través de sus APIs. Esta implementación llamada EuropeanaRS es software libre y su código se encuentra disponible en el siguiente repositorio: http://github.com/agordillo/EuropeanaRS. Además del propio SR, EuropeanaRS cuenta con una interfaz gráfica donde se ofrecen varios servicios a los usuarios: búsqueda y visionado de OAs, lista de favoritos, edición de perfil de usuario, etc. La Fig. 3 muestra cómo se visualiza un OA de Europeana y sus correspondientes recomendaciones en el portal web de EuropeanaRS. Los usuarios pueden acceder a EuropeanaRS de forma anónima, registrándose, o iniciando sesión con sus credenciales de Europeana. En este último caso se importa su perfil de usuario incluyendo su lista de favoritos.

\section{1) Casos de uso}

En este segundo escenario existen dos situaciones en las que se recomiendan OAs a los usuarios. La primera se da en la página principal del portal de EuropeanaRS donde se muestra una lista de sugerencias. Por otro lado, cuando un usuario accede a un OA se muestran recomendaciones mediante un carrusel de imágenes tal y como ilustra la Fig. 3. Ambas situaciones se dan para usuarios identificados y anónimos.

\section{2) Objetos de aprendizaje}

Los OAs de Europeana están etiquetados con metadatos conformes al esquema EDM [42]. Para esta implementación se usó un subconjunto de OAs compuesto por 12.380 números de periódicos históricos digitalizados procedentes de diversos países y años. La Fig. 3 muestra uno de estos recursos. Los OAs se modelaron mediante las propiedades título (tipo texto, peso 0,2), descripción (texto, 0,15), idioma (vocabulario, 0,5) y año de publicación (fecha, 0,15$)$.

\section{3) Perfiles de usuario}

Los usuarios se modelaron mediante su idioma (tipo vocabulario, peso 0,5$)$ y una lista con OAs que les interesaron en el pasado (peso 0,5). En esta ocasión, para usuarios registrados se emplea como lista los OAs añadidos a favoritos, y para usuarios anónimos se crea una lista virtual con los últimos recursos accedidos. El SR toma en consideración 2 OAs al azar de los últimos 5 . 


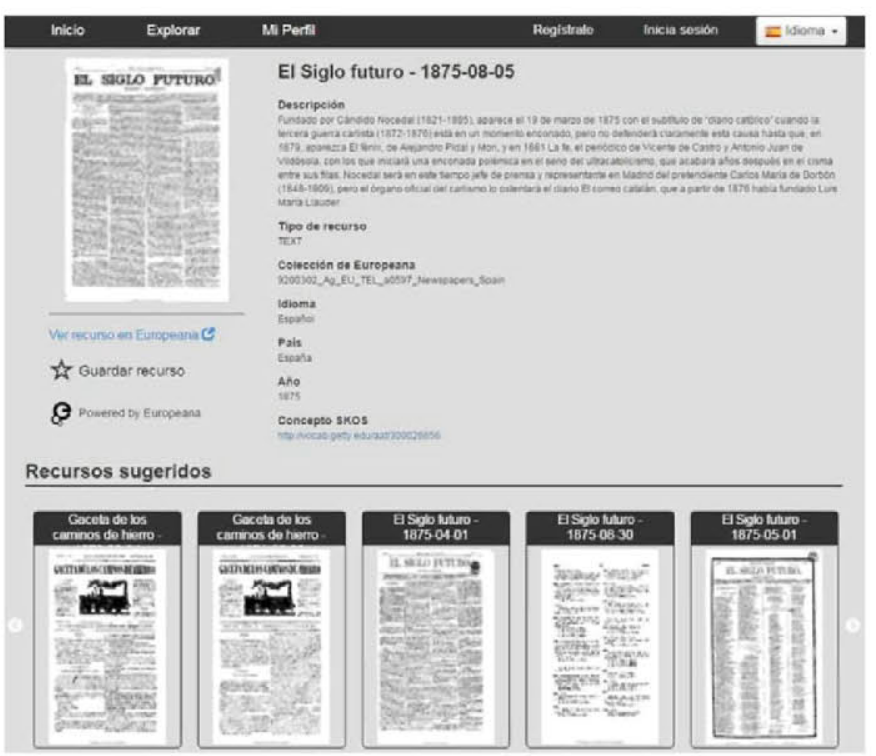

Figura 3. Objeto de Aprendizaje de Europeana en el portal EuropeanaRS.

\section{4) Puntuaciones de calidad y popularidad}

Europeana no proporciona medidas de calidad pedagógica de los OAs, ni tampoco ofrece puntuaciones de popularidad o estadísticas de uso de los mismos. Por estos motivos no se usaron criterios de calidad ni popularidad en este escenario.

\section{5) Proceso de recomendación}

En el paso de preselección se establecieron dos filtros: idioma y tipo de recurso. Estos filtros funcionan de manera análoga a los del escenario de ViSH.

A diferencia del escenario anterior, en EuropeanaRS los usuarios pueden modificar desde su perfil de usuario los ajustes del SR, incluyendo los pesos utilizados en el paso 2 y los umbrales de todos los filtros. Los pesos utilizados por defecto se muestran en la Tabla II. Los umbrales de los filtros tienen un valor inicial de 0 .

Las recomendaciones se muestran en forma de carruseles de imágenes tal y como muestra la Fig. 3. No obstante, el SR ofrece una API para aplicaciones externas que quieran generar recomendaciones de OAs de Europeana, y en este caso la forma de visualización dependería de la aplicación en cuestión. En este escenario el SR se nutre de las listas de OAs favoritos de los usuarios. Estas listas pueden ser construidas dentro de EuropeanaRS o bien pueden ser importadas de Europeana. Para usuarios anónimos esta lista se genera dinámicamente a medida que visitan recursos.

TABLA II

PESOS DEL SISTEMA DE RECOMENDACIÓN DE EUROPEANA

\begin{tabular}{|l|c|c|c|c|}
\hline \multirow{2}{*}{\begin{tabular}{c}
\multirow{2}{*}{$\begin{array}{c}\text { Criterios de } \\
\text { recomendación }\end{array}$} \\
\cline { 2 - 5 }
\end{tabular}} & \multicolumn{2}{|c|}{ Pesos por defecto usados en Europeana } \\
\cline { 2 - 5 } & Con $O A$ & Sin $O A$ & \multicolumn{2}{c|}{$\begin{array}{c}\text { Sin Usuario } \\
\text { (con Perfil Virtual) }\end{array}$} \\
\hline $\begin{array}{l}\text { Similitud con } O A \\
\text { de usuario }\end{array}$ & 0,5 & 1 & 0,5 & Sin $O A$ \\
\hline $\begin{array}{l}\text { Similitud con OA } \\
\text { contextual }\end{array}$ & 0,5 & - & 0,5 & 1 \\
\hline Calidad & 0 & 0 & 0 & 0 \\
\hline
\end{tabular}

\begin{tabular}{|l|c|c|c|c|}
\hline \multirow{2}{*}{$\begin{array}{c}\text { Criterios de } \\
\text { recomendación }\end{array}$} & \multicolumn{3}{|c|}{ Pesos por defecto usados en Europeana } \\
\cline { 2 - 5 } & \multicolumn{2}{|c|}{ Con Usuario } & \multicolumn{2}{c|}{$\begin{array}{c}\text { Sin Usuario } \\
\text { (con Perfil Virtual) }\end{array}$} \\
\cline { 2 - 5 } & Con $O A$ & Sin $O A$ & Con $O A$ & Sin $O A$ \\
\hline Popularidad & 0 & 0 & 0 & 0 \\
\hline
\end{tabular}

\section{EVALUACIÓN}

Esta sección expone el estudio de evaluación realizado y los resultados obtenidos.

\section{A. Estudio de evaluación}

En este estudio participaron 40 usuarios (30 hombres y 10 mujeres, con edades comprendidas entre los 22 y los 60 años), 20 evaluaron el SR de ViSH y 20 el de Europeana. Los usuarios realizaron la evaluación en línea desde sus propios equipos siguiendo una guía. Esta guía solicitaba a los participantes completar los siguientes pasos:

1. Registro en la plataforma web correspondiente: ViSH o EuropeanaRS.

2. Seguimiento de un tour guiado por el portal web para aprender a utilizar los servicios principales de la plataforma: búsqueda y visionado de OAs, sistema de favoritos, perfil de usuario, recomendaciones, etc.

3. Realización de 4 tareas. Uso libre de la plataforma.

4. Buscar al menos 5 OAs relevantes para el usuario y añadirlos a su lista de favoritos.

5. Rellenar un cuestionario de evaluación.

6. En este paso al usuario se le presenta una lista de recomendaciones con 12 OAs ordenados de forma aleatoria. El usuario debe valorar la relevancia que tiene cada OA para él mediante una escala Likert de 5 puntos.

7. Por último, se muestra al usuario un OA elegido al azar entre los que añadió a favoritos, junto con una nueva lista de recomendaciones con 12 OAs ordenados aleatoriamente. El usuario debe valorar, para esta situación concreta, la relevancia que tiene cada OA para él empleando la misma escala que en el paso anterior.

\section{B. Precisión del recomendador}

La precisión se evaluó mediante una validación cruzada dejando uno fuera (Leave-one-out cross-validation). En este análisis se consideraron los OAs pertenecientes a la listas de favoritos de los usuarios. Para cada usuario, se midió con qué frecuencia el OA dejado fuera aparecería en las primeras $\mathrm{N}$ recomendaciones (Top $N$ ), para $\mathrm{N}$ igual a $1,5,10$ y 20 . Es decir, se midió para cada usuario con qué frecuencia un OA de sus favoritos era recomendado en base su perfil de usuario sin tener en cuenta dicho OA.

En ViSH se hizo uso de toda la información disponible en la base de datos considerando 950 usuarios que tenían en sus listas de favoritos una media de 3,8 OAs. Los usuarios sin favoritos no fueron tenidos en cuenta. La cantidad total de OAs en ViSH en el momento del estudio era 9.479.

En la evaluación del SR de Europeana se utilizaron los 20 usuarios participantes en el estudio de evaluación, que tenían en sus listas de favoritos una media de 5,25 OAs. La cantidad total de OAs en EuropeanaRS en el momento del estudio era 
12.380. Los resultados del análisis se muestran en la Fig. 4.

La precisión de un sistema que recomendase OAs al azar sería inferior al $0,25 \%$ en el mejor de los casos $(\mathrm{N}=20)$ para ambos escenarios. Esta precisión es notablemente superada por los SRs implementados alcanzando para $\mathrm{N}=20$ un valor de $33,5 \%$ en ViSH y $24,9 \%$ en Europeana.

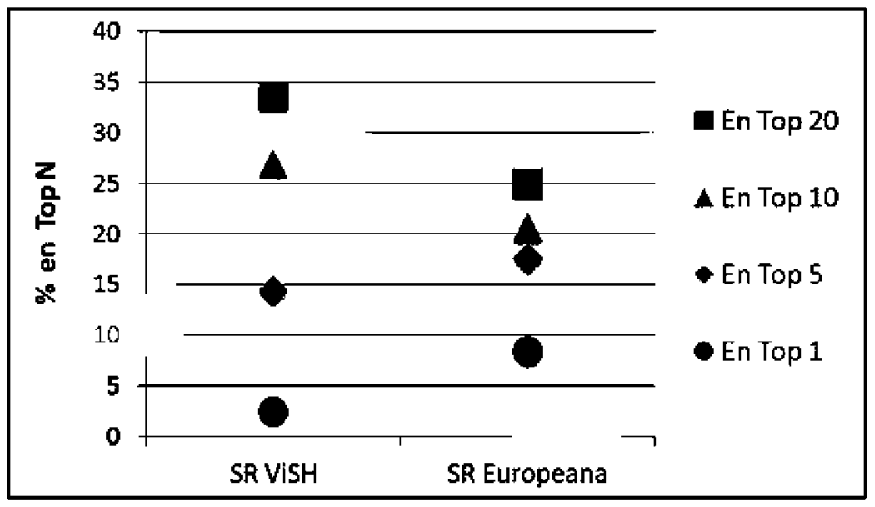

Figura 4. Precisión: resultados del análisis Leave-one-out

\section{Utilidad de las recomendaciones}

En el estudio de evaluación se les presentaron a los usuarios listas con recomendaciones en dos situaciones diferentes. En la primera los usuarios recibieron la lista como recomendaciones para su usuario en particular. En la segunda situación se les mostró a los usuarios un OA concreto acompañado de la lista. Cada una de estas situaciones se corresponde con uno de los casos de uso principales del modelo: recomendar $O A s$ a un usuario particular y recomendar a un usuario OAs similares a otro. Las listas se construyeron concatenando una lista de 6 OAs generada por el SR y otra lista con 6 OAs elegidos al azar. De este modo, las listas resultantes tenían 12 OAs dispuestos al azar, de los cuales 6 eran recomendaciones reales generadas por el SR, y los otros 6 eran OAs elegidos al azar entre todos los del repositorio. Los usuarios valoraron la relevancia de cada uno de los OAs sugeridos usando una escala Likert de 5 puntos. Para medir la utilidad de las recomendaciones generadas por el SR y las aleatorias usamos la métrica R-Score [43] normalizada. Esta métrica asume que el valor de las recomendaciones decrece de forma exponencial en función de su posición en la lista. De este modo, la utilidad de una lista de recomendaciones en una escala $0-1$ viene dada por:

$$
R(L)=\frac{1}{U} \sum_{u=1}^{U} \frac{\sum_{j=1}^{N} \frac{\max \left(U_{u j}-d, 0\right)}{\frac{j-1}{2 \alpha-1}}}{\sum_{j=1}^{N} \frac{\frac{5-d}{j-1}}{2 \frac{j-1}{\alpha-1}}}
$$

Donde $\mathrm{U}$ es el número de usuarios que han valorado los $\mathrm{N}$ elementos de la lista $\mathrm{L}, \mathrm{U}_{\mathrm{uj}}$ es la valoración que el usuario u-ésimo le ha dado al elemento situado en la posición j de la lista, d es un umbral ajustable que determina la valoración mínima que ha de alcanzar un elemento para ser considerado útil, y $\alpha$ es el parámetro de vida que controla el decrecimiento exponencial de los valores en función de la posición. En nuestro estudio establecimos el umbral d a 2 y el parámetro $\alpha$ a 3,5. La tabla III muestra las puntuaciones de utilidad calculadas mediante esta métrica para las listas generadas por los SRs (columna $S R$ ) y para las listas de OAs aleatorios (columna A) en los dos casos y escenarios abordados. La utilidad de las recomendaciones generadas por los SRs alcanzó valoraciones elevadas (superiores a $0,72 / 1$ ) superando notablemente la de las recomendaciones realizadas al azar.

TABLA III

UTLIDAD DE LAS RECOMENDACIONES (MÉTRICA R-SCORE)

\begin{tabular}{|c|c|c|c|c|c|c|c|}
\hline \multicolumn{4}{|c|}{ ViSH } & \multicolumn{4}{c|}{ Europeana } \\
\hline \multicolumn{2}{|c|}{ Caso I } & \multicolumn{2}{c|}{ Caso II } & \multicolumn{2}{c|}{ Caso I } & \multicolumn{2}{c|}{ Caso II } \\
\hline$S R$ & $A$ & $S R$ & $A$ & $S R$ & $A$ & $S R$ & $A$ \\
\hline 0,84 & 0,22 & 0,9 & 0,09 & 0,79 & 0,2 & 0,72 & 0,14 \\
\hline
\end{tabular}

\section{Usabilidad y satisfacción de los usuarios}

Estos aspectos de los SRs fueron evaluados a partir de un cuestionario que incluía la escala SUS (System Usability Scale) [44] y 14 preguntas basadas en el modelo ResQue [45]. SUS evalúa la facilidad de uso de un sistema a través de un cuestionario de 10 preguntas tipo Likert de 5 puntos arrojando una puntuación global en una escala 0-100. El modelo ResQue tiene el objetivo de evaluar diferentes aspectos de un SR como su interfaz e interacción, aceptación, o la satisfacción percibida por los usuarios. Las preguntas de este modelo son de tipo Likert de 5 puntos. El cuestionario fue rellenado por todos los participantes del estudio de evaluación $(\mathrm{n}=20)$. En el caso de ViSH, el cuestionario fue completado también por 30 usuarios habituales de la plataforma $(\mathrm{n}=50)$. Los resultados se muestran en la tabla IV. Estos resultados mostraron una alta usabilidad (puntuación SUS 84/100), así como una buena aceptación de los SRs por parte de los usuarios en términos de interfaz, interacción, satisfacción y utilidad. Todas las preguntas del modelo ResQue obtuvieron puntuaciones superiores a 3,2 en una escala $1-5$, con un promedio superior a 4. Si bien la puntuación SUS fue igual para ambos escenarios, las puntuaciones ResQue obtenidas en ViSH ( 4,3 de media) fueron sensiblemente superiores a las obtenidas para el SR de Europeana $(3,8)$.

TABLA IV

RESULTADOS DEL CUESTIONARIO DE EVALUACIÓN

\begin{tabular}{|c|c|c|}
\hline V (SR ViSH, $\mathbf{n = 5 0 ) , ~ E ~ ( S R ~ E u r o p e a n a , ~} n=20)$ & $\mathbf{V}$ & $\mathbf{E}$ \\
\hline Puntuación global SUS (escala 0-100) & 84 & 84 \\
\hline \multicolumn{3}{|l|}{ Preguntas ResQue (escala 1-5) } \\
\hline Los recursos recomendados encajan con mis intereses. & 4,4 & 4,1 \\
\hline $\begin{array}{l}\text { Algunos de los recursos recomendados me resultan } \\
\text { familiares. }\end{array}$ & 4,2 & 3,8 \\
\hline Los recursos recomendados son atractivos. & 4,2 & 3,9 \\
\hline Los recursos recomendados son nuevos e interesantes. & 3,9 & 3,6 \\
\hline Los recursos recomendados son diversos. & 3,9 & 3,2 \\
\hline Entendí por qué los recursos fueron recomendados para mí. & 4,1 & 3,9 \\
\hline $\begin{array}{l}\text { El diseño de la interfaz de recomendación es atractivo y } \\
\text { adecuado. }\end{array}$ & 4,6 & 3,9 \\
\hline Encontré fácilmente los recursos recomendados. & 4,7 & 4,5 \\
\hline $\begin{array}{l}\text { Fui capaz de aprovechar las ventajas del recomendador } \\
\text { rápidamente. }\end{array}$ & 4,4 & 4,1 \\
\hline $\begin{array}{l}\text { Los recursos recomendados me ayudaron de manera } \\
\text { efectiva a encontrar el objeto de aprendizaje ideal. }\end{array}$ & 4,1 & 3,7 \\
\hline
\end{tabular}




\begin{tabular}{|c|c|c|c|c|c|c|}
\hline \multicolumn{5}{|c|}{$\begin{array}{l}\text { Me siento respaldado para encontrar lo que quiero con la } \\
\text { ayuda del recomendador. }\end{array}$} & 4,2 & 3,8 \\
\hline \multicolumn{5}{|c|}{ En general, estoy satisfecho con el recomendador. } & 4,5 & 3,8 \\
\hline \multicolumn{5}{|c|}{$\begin{array}{l}\text { Estoy seguro de que me van a gustar los recursos } \\
\text { recomendados para mí. }\end{array}$} & 4,1 & 3,5 \\
\hline \multicolumn{5}{|c|}{ Voy a utilizar este tipo de recomendador con frecuencia. } & 4,2 & 3,8 \\
\hline \multicolumn{7}{|c|}{ ¿Cuál es su opinión general sobre el Sistema de Recomendación? } \\
\hline & Excelente & Buena & Neutral & Mala & \multicolumn{2}{|c|}{ Horrible } \\
\hline $\mathrm{V}$ & $48 \%$ & $44 \%$ & $8 \%$ & $0 \%$ & \multicolumn{2}{|c|}{$0 \%$} \\
\hline $\mathrm{E}$ & $30 \%$ & $50 \%$ & $15 \%$ & $5 \%$ & \multicolumn{2}{|c|}{$0 \%$} \\
\hline
\end{tabular}

\section{E. Test $A / B$}

Finalmente, se realizó un test $\mathrm{A} / \mathrm{B}$ en la plataforma ViSH durante 6 meses para evaluar el SR en un contexto totalmente real. En este test comparamos el SR desarrollado para ViSH siguiendo el modelo presentado con un sistema que realiza recomendaciones de forma aleatoria. Cuando un usuario llega a la última diapositiva de una Presentación Interactiva de ViSH puede pedir recomendaciones pulsando el botón de 'Siguiente diapositiva'. En caso de hacerlo se despliega un nuevo panel con 6 OAs recomendados. El usuario puede entonces acceder a cualquiera de los OAs o cerrar el panel rechazando las sugerencias. Esta situación fue la elegida para el test $\mathrm{A} / \mathrm{B}$ por ser la única en la que se produce una aceptación o rechazo explícito de las recomendaciones. Durante los 6 meses que duró el test, en torno al 50\% de las recomendaciones mostradas en esta situación fueron generadas por el SR de ViSH y el resto fueron generadas por un sistema que recomendaba OAs aleatoriamente. En este periodo se generaron un total de 8.979 conjuntos de recomendaciones de 6 OAs. Los sistemas fueron comparados en cuanto al ratio de aceptación de las recomendaciones y la calidad y tiempo de interacción con los usuarios de los OAs cuyas recomendaciones fueron aceptadas. La tabla $\mathrm{V}$ muestra los resultados del test $\mathrm{A} / \mathrm{B}$. El ratio de aceptación del SR $(27,4 \%)$ fue notablemente superior al logrado con las recomendaciones aleatorias $(12,9 \%)$. Esta diferencia es estadísticamente significativa (chi-cuadrado $=856, \mathrm{p}<0,0001$ ). En cuanto a la calidad y tiempo de interacción con usuarios el $\mathrm{SR}(\mathrm{Q}=7,04, \mathrm{t}=161,9)$ también superó de forma notable a las recomendaciones aleatorias $(\mathrm{Q}=5,49, \mathrm{t}=62,5)$, siendo estas diferencias estadísticamente significativas $(p<0,0001)$.

TABLA V

RESULTADOS DEL TEST A/B EN VISH

\begin{tabular}{|c|c|c|c|c|c|}
\hline Recomendaciones & \multicolumn{2}{|c|}{ SR ViSH } & \multicolumn{2}{|c|}{ Aleatorias } & \multirow{2}{*}{$\begin{array}{l}\text { Chi-cuadrado } \\
\left(X^{2}\right)\end{array}$} \\
\hline Totales & \multicolumn{2}{|c|}{4.591} & \multicolumn{2}{|c|}{4.388} & \\
\hline Aceptadas & \multicolumn{2}{|c|}{$1.258(27,4 \%)$} & \multicolumn{2}{|c|}{$567(12,9 \%)$} & $\begin{array}{c}856 \\
(p<0,0001)\end{array}$ \\
\hline $\begin{array}{c}\text { OAs } \\
\text { aceptados }\end{array}$ & M & $\mathrm{DE}$ & M & DE & $\begin{array}{c}\text { Test-t } \\
\text { p-valor }\end{array}$ \\
\hline Calidad (Q) & 7,04 & 1,53 & 5,49 & 1,44 & $<0,0001$ \\
\hline $\begin{array}{c}\text { Tiempo de } \\
\text { interacción (seg) }\end{array}$ & 161,9 & 231,6 & 62,5 & 162,6 & $<0,0001$ \\
\hline
\end{tabular}

\section{CONCLUSIONES}

Este artículo presenta un modelo de recomendación híbrida para Repositorios de Objetos de Aprendizaje (ROAs) que combina técnicas de recomendación basada en contenido, demográfica y sensible al contexto, junto con el uso de métricas de calidad $\mathrm{y}$ popularidad. El artículo también describe cómo se ha seguido el modelo para implementar dos Sistemas de Recomendación (SRs) para dos ROAs reales: ViSH y Europeana. Los resultados de las evaluaciones de los SRs mostraron una alta aceptación por parte de los usuarios en términos de utilidad, usabilidad, y satisfacción. En todas las pruebas realizadas los SRs superaron notablemente las prestaciones alcanzadas mediante recomendaciones aleatorias.

El presente trabajo contribuye a la literatura aportando la evaluación de un modelo de recomendación en dos escenarios reales. Este trabajo muestra que es posible diseñar modelos de recomendación flexibles, que soporten una amplia variedad de casos de uso, y que permitan implementar SRs en escenarios diferentes obteniendo buenos resultados, tanto técnicos como desde el punto de vista de los usuarios. El presente trabajo también expone a través de implementaciones reales los diferentes aspectos a los que debe adaptarse un modelo de recomendación cuando se aplica a un escenario concreto y estrategias para lograr esta adaptación.

Los resultados obtenidos por ambos SRs fueron buenos. El SR de ViSH tuvo mejores resultados que el de Europeana en utilidad y satisfacción, la usabilidad fue similar, y la precisión fue mayor en uno u otro dependiendo de la situación. Estas diferencias muestran la gran influencia del entorno sobre el $\mathrm{SR}$ en un escenario real. El factor más influyente es el conjunto de OAs utilizado, ya que afecta a aspectos como la diversidad o la capacidad de las recomendaciones para generar interés en los usuarios. Si un ROA no dispone de recursos abundantes, variados y atractivos, será complicado que un SR resulte efectivo en su escenario. Diferentes implementaciones de un modelo para un mismo escenario también pueden dar lugar a resultados distintos, ya que aspectos tales como la interfaz, la experiencia de usuario, el modelado de las entidades o las estrategias adoptadas para generar las recomendaciones tienen una gran influencia en el SR

Futuras investigaciones deberían estudiar la influencia en la efectividad de los SRs de diferentes factores tales como el conjunto de OAs del repositorio y su modelado, la estrategia seguida para construir el perfil de usuario o las métricas de calidad utilizadas. También resultaría interesante intentar mejorar el modelo introduciendo nuevos criterios basados en filtrado colaborativo o recomendación social, o adaptar el modelo para recomendar cursos completos o unidades didácticas en forma de secuencias de OAs.

\section{REFERENCIAS}

[1] J. Sinclair, M. Joy, J. Yin-Kim Yau, and S. Hagan, "A Practice-Oriented review of Learning Objects," IEEE Transactions on Learning Technologies, vol. 6, no. 2, pp. 177-192, 2013.

[2] D. A. Wiley, "Learning Object Design and Sequencing Theory," Brigham Young University, 2000

[3] IEEE LTSC, "Draft Standard for Learning Object Metadata (IEEE LOM)," 2002.

[4] "Dublin Core Metadata." [Online]. Available: http://www.dublincore.org.

[5] A. Baki and $\ddot{\mathrm{U}}$. Çakıroğlu, "Learning objects in high school mathematics 
classrooms: Implementation and evaluation," Computers \& Education, vol. 55 , no. 4 , pp. $1459-1469,2010$.

[6] R. Kay, "Exploring the use of web-based learning tools in secondary school classrooms," Interactive Learning Environments, 2012.

[7] A. M. Vasco, M. A. Amaral, N. S. Martins, and V. F. Bartholo, "Learning Objects to Suport the Teaching of Science," IEEE Latin America Transactions, vol. 9, no. 3, pp. 376-383, 2011.

[8] A. Tzikopoulos, N. Manouselis, and R. Vuorikari, "An Overview of Learning Object Repositories," in Learning Objects for Instruction: Design and Evaluation, 2007, pp. 29-55.

[9] P. Zervas, C. Alifragkis, and D. G. Sampson, "A quantitative analysis of learning object repositories as knowledge management systems," Knowledge Management \& E-Learning: An International Journal, vol. 6, no. 2, pp. 156-170, 2014.

[10] R. Kay, L. Knaack, and D. Petrarca, "Exploring Teachers Perceptions of Web-Based Learning Tools," Interdisciplinary Journal of E-Learning and Learning Objects, vol. 5, 2009.

[11] N. Y. Yen, T. K. Shih, L. R. Chao, and Q. Jin, "Ranking Metrics and Search Guidance for Learning Object Repository," IEEE Transactions on Learning Technologies, vol. 3, no. 3, pp. 250-264, 2010.

[12] K. Clements, J. Pawlowski, and N. Manouselis, "Open educational resources repositories literature review - Towards a comprehensive quality approaches framework," Computers in Human Behavior, vol. 51, part B, pp. 1098-1106, 2015.

[13] S. Fraihat and Q. Shambour, "A Framework of Semantic Recommender System for e-Learning," Journal of Software, vol. 10, no. 3, pp. 317-330, 2015.

[14] A. Ruiz-Iniesta, G. Jiménez-Díaz, and M. Gómez-Albarrán, "Personalización en Recomendadores Basados en Contenido y su Aplicación a Repositorios de Objetos de Aprendizaje," IEEE Revista Iberoamericana de Tecnologias del Aprendizaje, vol. 5, no. 1, pp. 31-38, 2010.

[15] C. Cechinel, M. Á. Sicilia, S. Sánchez-Alonso, and E. GarcíaBarriocanal, "Evaluating collaborative filtering recommendations inside large learning object repositories," Information Processing and Management, vol. 49, no. 1, pp. 34-50, 2013.

[16] A. Zapata, V. H. Menéndez, M. E. Prieto, and C. Romero, "A framework for recommendation in learning object repositories: An example of application in civil engineering," Advances in Engineering Software, vol. 56, pp. 1-14, 2013.

[17] H. Drachsler, K. Verbert, O. Santos, and N. Manouselis, "Panorama of recommender systems to support learning," in Recommender Systems Handbook, F. Ricci, L. Rokach, and B. Shapira, Eds. Springer US, 2015, pp. $421-451$

[18] N. Manouselis, H. Drachsler, R. Vuorikari, H. Hummel, and R. Koper, "Recommender Systems in Technology Enhanced Learning," in Recommender Systems Handbook, F. Ricci, L. Rokach, B. Shapira, and P. B. Kantor, Eds. Springer US, 2011, pp. 387-415.

[19] J. Picault, M. Ribière, D. Bonnefoy, and K. Mercer, "How to Get the Recommender Out of the Lab?," in Recommender Systems Handbook, F. Ricci, L. Rokach, B. Shapira, and P. B. Kantor, Eds. Springer US, 2011, pp. 333-365.

[20] F. Ricci, L. Rokach, and B. Shapira, "Introduction to Recommender Systems Handbook," in Recommender Systems Handbook, F. Ricci, L. Rokach, B. Shapira, and P. B. Kantor, Eds. Springer US, 2011, pp. 1-35.

[21] R. Burke, "Hybrid Web Recommender Systems," in The Adaptive Web, 2007, pp. 377-408.

[22] R. Burke, "Hybrid Recommender Systems: Survey and Experiments," User Modeling and User-Adapted Interaction, vol. 12, no. 4, pp. 331-370, 2002.

[23] K. Verbert, N. Manouselis, X. Ochoa, M. Wolpers, H. Drachsler, I. Bosnic, and E. Duval, "Context-aware recommender systems for learning: A survey and future challenges," IEEE Transactions on Learning Technologies, vol. 5, no. 4, pp. 318-335, 2012.

[24] D. Gallego, E. Barra, S. Aguirre, and G. Huecas, "A Model for Generating Proactive Context-Aware Recommendations in e-Learning Systems," in Proceedings of the 2012 Frontiers in Education Conference, 2012.

[25] D. Gallego, E. Barra, P. Rodriguez, and G. Huecas, "Incorporating proactivity to context-aware recommender systems for e-learning," in World Congress on Computer and Information Technology (WCCIT) 2013, 2013, pp. 1-6.

[26] P. Clough, A. Otegi, E. Agirre, and M. Hall, "Implementing Recommendations in the PATHS System," in Theory and Practice of Digital Libraries -- TPDL 2013 Selected Workshops, Springer
International Publishing, 2014, pp. 169-173.

[27] A. Otegi, E. Agirre, and P. Clough, "Personalised PageRank for Making Recommendations in Digital Cultural Heritage Collections," in IEEE/ACM Joint Conference on Digital Libraries (JCDL) 2014, 2014, pp. $49-52$.

[28] H. Imran, Q. Hoang, T. Chang, and S. Graf, "A Framework to Provide Personalization in Learning Management Systems through a Recommender System Approach," in Intelligent Information and Database Systems, Springer International Publishing, 2014, pp. $271-280$.

[29] F. M. Mendes Neto and A. F. A. Sales, "A Recommendation System for Ubiquitous Learning in the Context of Formal and Informal Education," IEEE Latin America Transactions, vol. 13, no. 4, pp. 1061-1067, 2015.

[30] F. M. Mendes Neto, A. A. L. da Costa, E. L. Sombra, J. D. C. Moreira, R. A. M. Valentim, J. J. Samper, R. P. C. do Nascimento, and C. D. Flores, "Content's Personalized Recommendation for Implementing Ubiquitous Learning in Health 2.0," IEEE Latin America Trasactions, vol. 12, no. 8, pp. 1515-1522, 2014.

[31] A. Corbi and D. Burgos, "Implementation of the recommendation model LIME in cognitive and visual interactive tutors from PSLC," IEEE Latin America Transactions, vol. 13, no. 2, pp. 516-522, 2015.

[32] M. P. O'Mahony and B. Smyth, "A recommender system for on-line course enrolment: an initial study," in Proceedings of the 2007 ACM conference on Recommender systems, 2007, pp. 133-136.

[33] T. L. Leacock and J. C. Nesbit, "A Framework for Evaluating the Quality of Multimedia Learning Resources," Educational Technology and Society, vol. 10, pp. 44-59, 2007.

[34] R. Kay, "Evaluating learning, design, and engagement in web-based learning tools (WBLTs): The WBLT Evaluation Scale," Computers in Human Behavior, vol. 27, no. 5, pp. 1849-1856, Sep. 2011.

[35] A. Gordillo, E. Barra, and J. Quemada, "Towards a Learning Object pedagogical quality metric based on the Learning Object Review Instrument," in Proceedings of the 2014 Frontiers in Education Conference, 2014.

[36] J. Sanz-Rodriguez, J. M. Dodero, and S. Sanchez-Alonso, "Ranking Learning Objects through Integration of Different Quality Indicators," IEEE Transactions on Learning Technologies, vol. 3, no. 4, pp. $358-363,2010$.

[37] S. Robertson, "Understanding inverse document frequency: on theoretical arguments for IDF," Journal of Documentation, vol. 60, no. 5 , pp. 503-520, 2004.

[38] E. Barra, A. Gordillo, and J. Quemada, "Virtual Science Hub: An Open Source Platform to Enrich Science Teaching," International Journal of Social, Education, Economics and Management Engineering, vol. 8, no. 3, 2014.

[39] A. Gordillo, E. Barra, and J. Quemada, "Facilitating the creation of interactive multi-device Learning Objects using an online authoring tool," in Proceedings of the 2014 Frontiers in Education Conference, 2014.

[40] G. Pavlidis and V. Sevetlidis, "Demystifying publishing to Europeana: A practical workflow for content providers," Scientific Culture, vol. 15, no. 1, pp. 1-7, 2015.

[41] Europeana Foundation, Transforming the world with culture (White Paper). 2015.

[42] Europeana Foundation, Europeana Data Model Primer. 2013.

[43] G. Shani and A. Gunawardana, "Evaluating Recommendation Systems," in Recommender Systems Handbook, F. Ricci, L. Rokach, B. Shapira, and P. B. Kantor, Eds. Springer US, 2011, pp. 257-297.

[44] J. Brooke, "This document: More Project information and further documents: SUS - A quick and dirty usability scale," in Usability evaluation in industry, 1996.

[45] P. Pu and L. Chen, "A User-Centric Evaluation Framework of Recommender Systems," in Proceedings of the fifth ACM conference on Recommender systems, 2011, pp. 157-164.

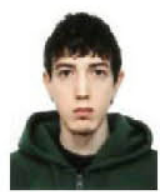

Aldo Gordillo se licenció en 2012 como Ingeniero de Telecomunicación en la Universidad Politécnica de Madrid, donde actualmente es candidato a doctor en Ingeniería de Sistemas Telemáticos. El ha trabajado como investigador e ingeniero web desde 2008. Sus intereses de investigación están en el campo del aprendizaje potenciado por la tecnología, con especial interés en objetos de aprendizaje. 
Enrique Barra recibió el título de doctor en Ingeniería de Sistemas Telemáticos por la Universidad Politécnica de Madrid en el año 2015. Actualmente trabaja como investigador en esta misma universidad. Sus intereses de investigación incluyen generación y distribución de contenidos educativos, videoconferencia y redes sociales.

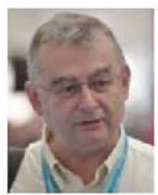

Juan Quemada es profesor en la Escuela Técnica Superior de Ingenieros de Telecomunicación de la Universidad Politécnica de Madrid (UPM). Es el coordinador del grupo de investigación Internet de Nueva Generación y el representante de la UPM en el WC3. Sus intereses de investigación se centran en arquitectura de aplicaciones colaborativas y sociales para Internet y la Web. 